PROCEEDINGS OF THE

AMERICAN MATHEMATICAL SOCIETY

Volume 136, Number 11, November 2008, Pages 4075-4078

S 0002-9939(08)09533-6

Article electronically published on June 2, 2008

\title{
FIRST EIGENVALUES OF GEOMETRIC OPERATORS UNDER THE RICCI FLOW
}

\author{
XIAODONG CAO \\ (Communicated by Chuu-Lian Terng)
}

\begin{abstract}
In this paper, we prove that the first eigenvalues of $-\Delta+c R(c \geq$ $\frac{1}{4}$ ) are nondecreasing under the Ricci flow. We also prove the monotonicity under the normalized Ricci flow for the cases $c=1 / 4$ and $r \leq 0$.
\end{abstract}

\section{First eigenvalue of $-\Delta+c R$}

Let $M$ be a closed Riemannian manifold, and $(M, \mathbf{g}(t))$ be a smooth solution to the Ricci flow equation

$$
\frac{\partial}{\partial t} \mathbf{g}_{i j}=-2 R_{i j}
$$

on $0 \leq t<T$. In Cao07, we prove that all eigenvalues $\lambda(t)$ of the operator $-\Delta+\frac{R}{2}$ are nondecreasing under the Ricci flow on manifolds with a nonnegative curvature operator. Assume $f=f(x, t)$ is the corresponding eigenfunction of $\lambda(t)$; that is,

$$
\left(-\Delta+\frac{R}{2}\right) f(x, t)=\lambda(t) f(x, t)
$$

and $\int_{M} f^{2} d \mu=1$. More generally, we define

$$
\lambda(f, t)=\int_{M}\left(-\Delta f+\frac{R}{2} f\right) f d \mu,
$$

where $f$ is a smooth function satisfying

$$
\frac{d}{d t}\left(\int_{M} f^{2} d \mu\right)=0, \int_{M} f^{2} d \mu=1 .
$$

We can then derive the monotonicity formula under the Ricci flow.

Theorem 1.1 (Cao07]). On a closed Riemannian manifold with a nonnegative curvature operator, the eigenvalues of the operator $-\Delta+\frac{R}{2}$ are nondecreasing under the Ricci flow. In particular,

$$
\frac{d}{d t} \lambda(f, t)=2 \int_{M} R_{i j} f_{i} f_{j} d \mu+\int_{M}|R c|^{2} f^{2} d \mu \geq 0 .
$$

In (1.2), when $\frac{d}{d t} \lambda(f, t)$ is evaluated at time $t, f$ is the corresponding eigenfunction of $\lambda(t)$.

Received by the editors October 5, 2007.

2000 Mathematics Subject Classification. Primary 58C40; Secondary 53C44.

This research was partially supported by an MSRI postdoctoral fellowship.

(C)2008 American Mathematical Society Reverts to public domain 28 years from publication 
Remark 1.2. Clearly, at time $t$, if $f$ is the eigenfunction of the eigenvalue $\lambda(t)$, then $\lambda(f, t)=\lambda(t)$. By the eigenvalue perturbation theory, we may assume that there is a $C^{1}$-family of smooth eigenvalues and eigenfunctions (for example, see [KL06, RS78 and $\left.\left.\mathrm{CCG}^{+} 07\right]\right)$. When $\lambda$ is the lowest eigenvalue, we can further assume that the corresponding eigenfunction $f$ is positive. Since the above formula does not depend on the particular evolution of $f, \frac{d}{d t} \lambda(t)=\frac{d}{d t} \lambda(f, t)$.

Remark 1.3. In Li07a, J. Li used the same technique to prove the monotonicity of the first eigenvalue of $-\Delta+\frac{1}{2} R$ under the Ricci flow without assuming a nonnegative curvature operator. A similar result appeared in the physics literature OSW05.

Remark 1.4. When $c=\frac{1}{4}$, the monotonicity of the first eigenvalue has been established by G. Perelman in Per02. The evolution of the first eigenvalue of the Laplace operator under the Ricci flow has been studied by L. Ma in [Ma06]. The evolution of the Yamabe constant under the Ricci flow has been studied by S.C. Chang and P. Lu in CL07.

In this paper, we shall study the first eigenvalues of operators $-\Delta+c R\left(c \geq \frac{1}{4}\right)$ without curvature assumption on the manifold. Our first result is the following theorem.

Theorem 1.5. Let $\left(M^{n}, \mathbf{g}(t)\right), t \in[0, T)$, be a solution of the Ricci flow on a closed Riemannian manifold $M^{n}$. Assume that $\lambda(t)$ is the lowest eigenvalue of $-\Delta+c R$ $\left(c \geq \frac{1}{4}\right), f=f(x, t)>0$ satisfies

$$
-\Delta f(x, t)+c R f(x, t)=\lambda(t) f(x, t)
$$

and $\int_{M} f^{2}(x, t) d \mu=1$. Then under the Ricci flow, we have

$$
\frac{d}{d t} \lambda(t)=\frac{1}{2} \int_{M}\left|R_{i j}+\nabla_{i} \nabla_{j} \varphi\right|^{2} e^{-\varphi} d \mu+\frac{4 c-1}{2} \int_{M}|R c|^{2} e^{-\varphi} d \mu \geq 0,
$$

where $e^{-\varphi}=f^{2}$.

Proof of Theorem 1.5. Let $\varphi$ be a function satisfying $e^{-\varphi(x)}=f^{2}(x)$. We proceed as in Cao07; we have

$$
\begin{aligned}
\frac{d}{d t} \lambda(t)= & \left(2 c-\frac{1}{2}\right) \int R_{i j} \nabla_{i} \varphi \nabla_{j} \varphi e^{-\varphi} d \mu \\
& -(2 c-1) \int R_{i j} \nabla_{i} \nabla_{j} \varphi e^{-\varphi} d \mu+2 c \int|R c|^{2} e^{-\varphi} d \mu .
\end{aligned}
$$

Integrating by parts and applying the Ricci formula, it follows that

$$
\int R_{i j} \nabla_{i} \nabla_{j} \varphi e^{-\varphi} d \mu=\int R_{i j} \nabla_{i} \varphi \nabla_{j} \varphi e^{-\varphi} d \mu-\frac{1}{2} \int R \Delta e^{-\varphi} d \mu
$$

and

$$
\begin{aligned}
\int R_{i j} \nabla_{i} \nabla_{j} \varphi e^{-\varphi} d \mu & +\int|\nabla \nabla \varphi|^{2} e^{-\varphi} d \mu \\
= & -\int \Delta e^{-\varphi}\left(\Delta \varphi+\frac{1}{2} R-\frac{1}{2}|\nabla \varphi|^{2}\right) d \mu \\
= & \left(2 c-\frac{1}{2}\right) \int R \Delta e^{-\varphi} d \mu .
\end{aligned}
$$


In the last step, we use

$$
2 \lambda(t)=\Delta \varphi+2 c R-\frac{1}{2}|\nabla \varphi|^{2} .
$$

Combining (1.5) and (1.6), we arrive at

$$
\int|\nabla \nabla \varphi|^{2} e^{-\varphi} d \mu=2 c \int R \Delta e^{-\varphi} d \mu-\int R_{i j} \nabla_{i} \varphi \nabla_{j} \varphi e^{-\varphi} d \mu .
$$

Plugging (1.7) into (1.4), we have

$$
\begin{aligned}
\frac{d}{d t} \lambda(t)= & \int R_{i j} \nabla_{i} \nabla_{j} \varphi e^{-\varphi} d \mu+2 c \int|R c|^{2} e^{-\varphi} d \mu \\
& +c \int R \triangle\left(e^{-\varphi}\right) d \mu-\frac{1}{2} \int R_{i j} \nabla_{i} \varphi \nabla_{j} \varphi e^{-\varphi} d \mu \\
= & \int R_{i j} \nabla_{i} \nabla_{j} \varphi e^{-\varphi} d \mu+2 c \int|R c|^{2} e^{-\varphi} d \mu+\frac{1}{2} \int|\nabla \nabla \varphi|^{2} e^{-\varphi} d \mu \\
= & \frac{1}{2} \int\left|R_{i j}+\nabla_{i} \nabla_{j} \varphi\right|^{2} e^{-\varphi} d \mu+\left(2 c-\frac{1}{2}\right) \int|R c|^{2} e^{-\varphi} d \mu \geq 0 .
\end{aligned}
$$

This proves the theorem as desired.

\section{First eigenvalue under the normalized Ricci Flow}

In this section, we derive the evolution of $\lambda(t)$ under the normalized Ricci flow equation

$$
\frac{\partial}{\partial t} \mathbf{g}_{i j}=-2 R_{i j}+\frac{2}{n} r \mathbf{g}_{i j}
$$

Here $r=\frac{\int_{M} R d \mu}{\int_{M} d \mu}$ is the average scalar curvature. It follows from Equation (1.3) that $\lambda \leq c r$. We now compute the derivative of the lowest eigenvalue of $-\Delta+c R$.

Theorem 2.1. Let $\left(M^{n}, \mathbf{g}(t)\right), t \in[0, T)$, be a solution of the normalized Ricci flow on a closed Riemannian manifold $M^{n}$. Assume that $\lambda(t)$ is the lowest eigenvalue of $-\Delta+c R\left(c \geq \frac{1}{4}\right)$ and that $f>0$ is the corresponding eigenfunction. Then under the normalized Ricci flow, we have

$$
\frac{d}{d t} \lambda(t)=-\frac{2 r \lambda}{n}+\frac{1}{2} \int_{M}\left|R_{i j}+\nabla_{i} \nabla_{j} \varphi\right|^{2} e^{-\varphi} d \mu+\frac{4 c-1}{2} \int_{M}|R c|^{2} e^{-\varphi} d \mu ;
$$

where $e^{-\varphi}=f^{2}$. Furthermore, if $c=\frac{1}{4}$ and $r \leq 0$, then

$$
\frac{d}{d t} \lambda(t)=\frac{2}{n} r\left(\lambda-\frac{r}{4}\right)+\frac{1}{2} \int_{M}\left|R_{i j}+\nabla_{i} \nabla_{j} \varphi-\frac{r}{n} g_{i j}\right|^{2} e^{-\varphi} d \mu \geq 0 .
$$

Remark 2.2. After we submitted our paper, J. Li suggested to us that (2.2) is true for all $c \geq 1 / 4$, with an additional nonnegative term

$$
\frac{4 c-1}{2} \int_{M}\left|R c-\frac{r}{n} g_{i j}\right|^{2} e^{-\varphi} d \mu ;
$$

see [Li07b] for a similar result.

Remark 2.3. As a consequence of the above monotonicity formula of $\lambda(t)$, we can prove that both compact steady and expanding Ricci breathers (cf. Ive93], [Per02]) must be trivial. Such results have been discussed by many authors (for example, see [ve93, Ham95], Ham88, Per02, Cao07 and Li07a, etc.).

When $M$ is a two-dimensional surface, $r$ is a constant. We have the following corollary. 
Corollary 2.4. Let $\left(M^{2}, \mathbf{g}(t)\right), t \in[0, T)$, be a solution of the normalized Ricci flow on a closed Riemannian surface $M^{2}$. Assume that $\lambda(t)$ is the lowest eigenvalue of $-\Delta+c R\left(c \geq \frac{1}{4}\right)$; we have $e^{r t} \lambda$ is nondecreasing under the normalized Ricci flow. Moreover, if $r \leq 0$, then $\lambda$ is nondecreasing.

\section{ACKNOWLEDGEMENTS}

The author would like to thank Dr. Junfang Li for bringing [Li07a] and Li07b] to his attention and for helpful discussions on this subject. He would also like to thank Professor Eric Woolgar for his interest and for pointing out the reference OSW05. He would like to thank Professor Duong H. Phong for his interest and encouragement. He also wants to thank MSRI for their generous support.

\section{REFERENCES}

[Cao07] Xiaodong Cao, Eigenvalues of $\left(-\Delta+\frac{R}{2}\right)$ on manifolds with nonnegative curvature operator, Math. Ann. 337 (2007), no. 2, 435-441. MR2262792 (2007g:53071)

$\left[\mathrm{CCG}^{+}\right.$07] Bennett Chow, Sun-Chin Chu, David Glickenstein, Christine Guenther, James Isenberg, Tom Ivey, Dan Knopf, Peng Lu, Feng Luo, and Lei Ni, The Ricci flow: techniques and applications. Part I, Geometric aspects. Mathematical Surveys and Monographs, vol. 135, American Mathematical Society, Providence, RI, 2007. MR2302600

[CL07] Shu-Cheng Chang and Peng Lu, Evolution of Yamabe constant under Ricci flow, Ann. Global Anal. Geom. 31 (2007), no. 2, 147-153. MR2326418

[Ham88] Richard S. Hamilton, The Ricci flow on surfaces, Mathematics and general relativity (Santa Cruz, CA, 1986), Amer. Math. Soc., Providence, RI, 1988, pp. 237-262. MR 89i:53029

[Ham95] , The formation of singularities in the Ricci flow, Surveys in differential geometry, Vol. II (Cambridge, MA, 1993), Internat. Press, Cambridge, MA, 1995, pp. 7-136. MR 97e:53075

[Ive93] Thomas Ivey, Ricci solitons on compact three-manifolds, Differential Geom. Appl. 3 (1993), no. 4, 301-307. MR.94j:53048

[KL06] Bruce Kleiner and John Lott, Notes on Perelman's papers, 2006.

[Li07a] Junfang Li, Eigenvalues and energy functionals with monotonicity formulae under Ricci flow, Math. Ann. 338 (2007), no. 4, 927-946. MR2317755(2008c:53068)

[Li07b] Monotonicity formulas under rescaled Ricci flow.

[Ma06] Li Ma, Eigenvalue monotonicity for the Ricci-Hamilton flow, Ann. Global Anal. Geom. 29 (2006), no. 3, 287-292. MR2248073 (2007c:53086)

[OSW05] T. Oliynyk, V. Suneeta, and E. Woolgar, Irreversibility of world-sheet renormalization group flow, Phys. Lett. B 610 (2005), no. 1-2, 115-121. MR.2118230 (2005h:81232)

[Per02] Grisha Perelman, The entropy formula for the Ricci flow and its geometric applications, 2002.

[RS78] Michael Reed and Barry Simon, Methods of modern mathematical physics. IV. Analysis of operators, Academic Press [Harcourt Brace Jovanovich Publishers], New York, 1978. MR.0493421(58:12429c)

Department of Mathematics, Cornell University, Ithaca, New York 14853-4201

E-mail address: cao@math.cornell.edu 